\title{
Aksel Tibet, un collègue à Porsuk et de plus un ami très cher
}

\section{Françoise Laroche-Traunecker}

\section{(2) OpenEdition}

\section{Journals}

Édition électronique

URL : https://journals.openedition.org/anatoliaantiqua/1226

DOI : 10.4000/anatoliaantiqua. 1226

\section{Éditeur}

IFEA

Édition imprimée

Date de publication : 31 décembre 2019

Pagination : 9-11

ISBN : 978-2-36245-080-8

ISSN : 1018-1946

\section{Référence électronique}

Françoise Laroche-Traunecker, «Aksel Tibet, un collègue à Porsuk et de plus un ami très cher »,

Anatolia Antiqua [En ligne], XXVII | 2019, mis en ligne le 26 février 2020, consulté le 17 mars 2022. URL http://journals.openedition.org/anatoliaantiqua/1226; DOI : https://doi.org/10.4000/anatoliaantiqua. 1226 


\title{
ANATOLIA ANTIQUA ESKI ANADOLU
}

\author{
XXVII
}

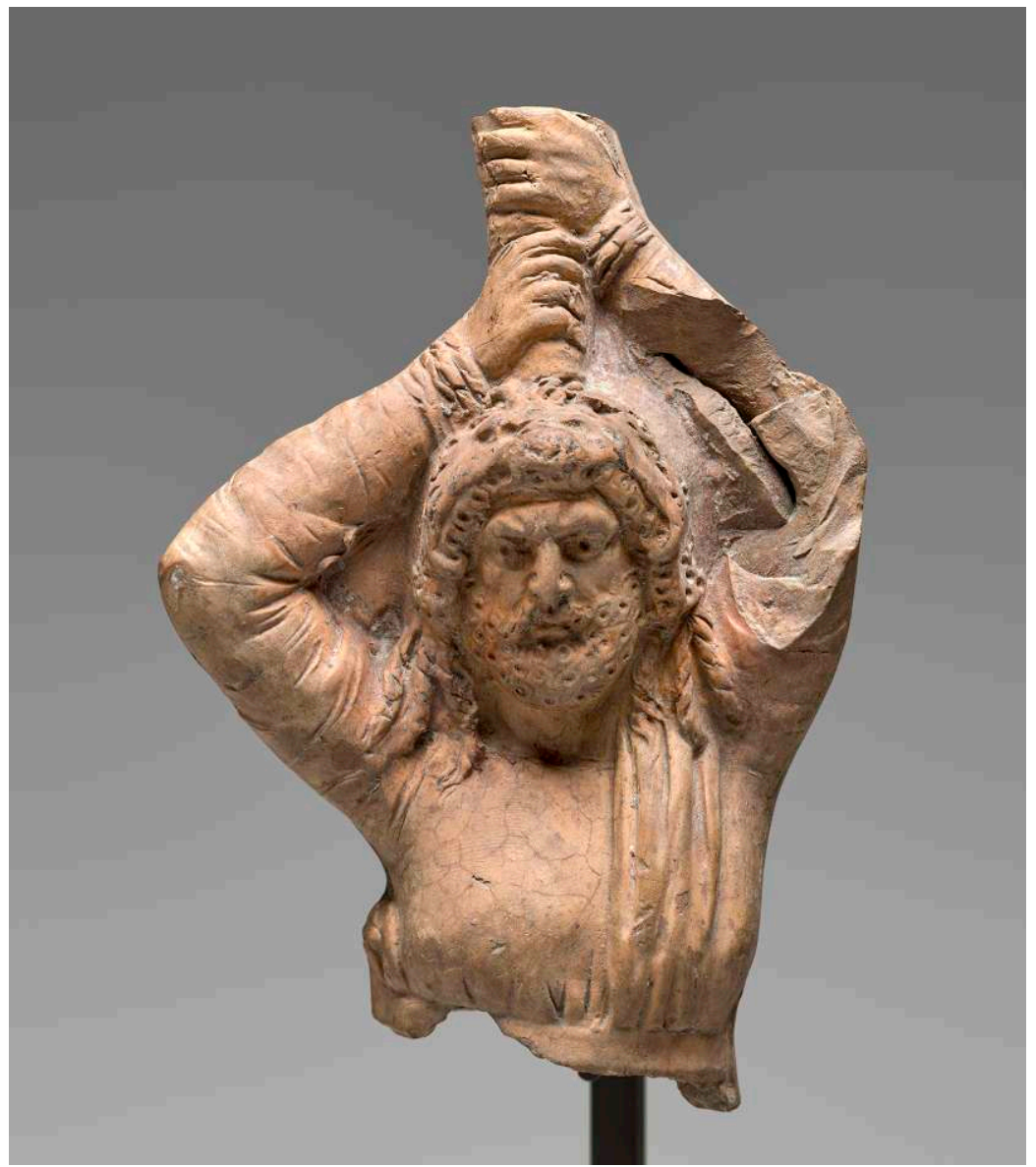

INSTITUT FRANÇAIS D'ETUDES ANATOLIENNES GEORGES-DUMEZIL CNRS USR 3131

DE BOCCARD 


\section{ANATOLIA ANTIQUA ESKI ANADOLU XXVII}

Recueil de travaux publiés par

l'Institut Français d'Etudes Anatoliennes Georges-Dumézil Istanbul

édite par

Deniz GENCEOLU et Martin GODON

\section{OFFPRINT / AYRIBASIM}

INSTITUT FRANÇAIS D'ETUDES ANATOLIENNES GEORGES - DUMEZIL CNRS USR 3131

DE BOCCARD Edition - Diffusion

11, rue de Médicis

75006 Paris 


\section{Comité de lecture}

Mme Catherine ABADIE-REYNAL, Université Lumière-Lyon 2.

Mme Nur BALKAN-ATLI, Université d'Istanbul.

M. Dominique BEYER, Université de Strasbourg.

M. Wolfgang BLÜMEL, Université de Cologne.

Mme Isabella CANEVA, Université de Salento (Lecce). Mme Marie-Claire CAUVIN, CNRS.

M. Jacques des COURTILS, Université Michel de Montaigne Bordeaux 3.

M. Alain DAVESNE, Université d'Orléans.

Mme Roberta FABIANI, Université de Pérouse. Mme Véronique FRANÇOIS, CNRS.

Mme Marcella FRANGIPANE, Université de Rome, "La Sapienza". Mme Marie-Henriette GATES, Université Bilkent (Ankara).

M. Harald HAUPTMANN, Université de Heidelberg.

M. Wilfried HELD, Université de Marburg.

M. Jean-Louis HUOT, Université Paris 1 Panthéon-Sorbonne.

M. Francis JOANNES, Université Paris 8.

Mme Christine KEPINSKI, CNRS.

M. Koray KONUK, CNRS.

Mme Catherine KUZUCUOĞLU, CNRS.

M. René LEBRUN, Université catholique de Louvain.

M. Christian LEROY, Université Paris 1 Panthéon-Sorbonne.

Mme Vasilica LUNGU, Institute of South-Eastern European Studies, Académie Roumaine.

Mme Raffaella PIEROBON-BENOIT, Université de Naples.

Mme Catherine MARRO, CNRS.

M. Philipp NIEWÖHNER, Université d'Oxford.

M. Alistair NORTHEDGE, Université Paris IV-Sorbonne.

Mme Mihriban ÖZBAŞARAN, Université d'Istanbul.

Mme Christine ÖZGAN, Université Mimar Sinan des Beaux-Arts (Istanbul).

M. Francis PROST, Ecole Normale Supérieure (Paris).

M. Cemal PULAK, Université de Texas A\&M.

M. Jean-Pierre SODINI, Académie des Inscriptions et Belles-Lettres (Paris).

M. Rahmi Hüseyin ÜNAL, Université d'Ege (İzmir).

Ce volume a été composé par les soins

de l'Institut Français d'Etudes Anatoliennes-Georges Dumézil

et Zero Prod. Ltd. Abdullah Sok. 17 Taksim 34433 Beyoğlu, Istanbul, Turquie, et imprimé par Matsis Matbaa Hizmetleri San. Ve Tic. Ltd. Şti.

La publication a pu en être réalisée grâce au concours financier

du Ministère de l'Europe et des Affaires étrangères, et du Centre National de la Recherche Scientifique.

CC 2019, Institut Français d'Etudes Anatoliennes Georges-Dumézil - Istanbul

La loi du 11 mars 1957 n'autorisant, aux termes des alinéas 2 et 3 de l'article 41, d'une part, que les "copies ou reproductions strictement réservées à l'usage privé du copiste et non destinées à une utilisation collective" et, d'autre part, que les analyses et les courtes citations dans un but d'exemple et d'illustration, "toute" représentation ou reproduction intégrale, ou partielle, faite sans le consentement de l'auteur ou de ses ayants droit ou ayants cause, est illicite (alinéa 1er de l'article 40).

Cette représentation ou reproduction, par quelque procédé que ce soit, constituerait donc une contefaçon sanctionnée par les articles 425 et suivants du Code Pénal. 


\section{TABLE DES MATIERES}

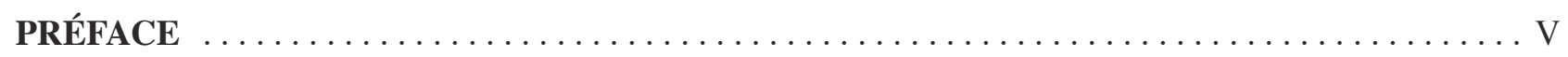

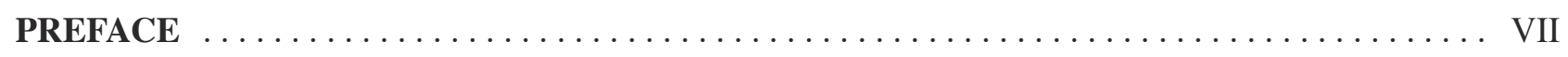

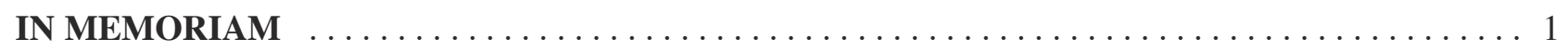

Burçin ERDOĞU et Özlem ÇEVIK

A Comparative Study of the Sixth Millennium BC Marble Bracelets from Ulucak and Uğurlu . . . . . 29

Martin GODON et Ozan ÖZBUDAK

Early Chalcolithic Pottery from Tepecik-Çiftlik and Gelveri, Central Anatolia:

From Local to Supra-Regional Ceramic Style $\ldots \ldots \ldots \ldots \ldots \ldots \ldots \ldots \ldots \ldots \ldots \ldots \ldots \ldots \ldots \ldots \ldots \ldots \ldots$

Vladimir SHELESTIN

On the Origin of Küçükçekmece Iron Figurines $\ldots \ldots \ldots \ldots \ldots \ldots \ldots \ldots \ldots \ldots \ldots \ldots \ldots \ldots \ldots \ldots \ldots \ldots \ldots$

Akın ERSOY et Ludovic LAUGIER

Sculptures grecques et romaines de Smyrne, découvertes récentes $\ldots \ldots \ldots \ldots \ldots \ldots \ldots \ldots \ldots 7$

Élisabeth GOUSSARD, Raphaëlle CHEVALLIER, Olivier HENRY et Christophe BOST

Labraunda 2018, Étude d'un poids décoré et d'une couronne :

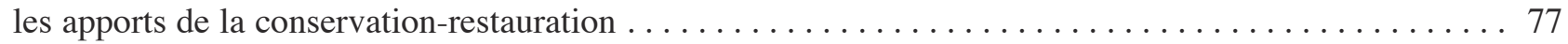

Isabelle HASSELIN ROUS

Les figurines en terre cuite de Tarse en Cilicie à l'époque romaine impériale $\ldots \ldots \ldots \ldots \ldots \ldots$

İnci TÜRKOĞLU

Early Byzantine Structure at Gerenkuyu Mevkii of Yal1-Bodrum . . . . . . . . . . . . . . . . . 109

Anca DAN, Sait BAŞARAN, Helmut BRÜCKNER, Ercan ERKUL, Anna PINT,

Wolfgang RABBEL, Lyudmila SHUMILOVSKIKH, Dennis WILKEN and Tina WUNDERLICH

Ainos in Thrace: Research Perspectives in Historical Geography and Geoarchaeology . . . . . . . 127

Hülya ATAŞCIOĞLU AYKUL, M. Baha TANMAN, Miguel Ángel ESCOBAR-CLARÓS

A Note on the Turkish Lot III / 1891 from the Bab el-Gasus Cache (Egypt), kept at the Istanbul

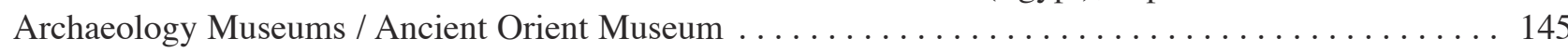

\section{CHRONIQUES DES TRAVAUX ARCHEOLOGIQUES EN TURQUIE 2018}

Kenan IŞIK, Bülent GENÇ, Vedat TIMMUR et Rıfat KUVANÇ

Two Column Bases From Mawan in the Hakkâri Province (Southeast Corner of Turkey):

A New Achaemenid Center? 


\section{Çiğdem MANER}

Preliminary Report on the Sixth Season of the Konya Ereğli, Karapınar, Halkapınar and

Emirgazi Survey Project (KEYAR) 2018 .

Erkan KONYAR, Bülent GENÇ, Can AVCI et Armağan TAN

Excavations at the Old City, Fortress, and Mound of Van: Work in 2018

Olivier HENRY et J. BLID, Chr. BOST, N. CARLESS-UNWIN, R. CHEVALLIER, G. ÇIMEN,

A. EYIGÖR, A. FREJMAN, E. GOUSSARD, V. LUNGU, A. SITZ, A. MUSAT-STREINU,

B. VERGNAUD

Labraunda 2018

M. SEYER, A. DOLEA, P. M. BES, D. Zs. SCHWARCZ, S. BAYBO, A. K. L. LEUNG,

U. QUATEMBER, M. WÖRRLE, H. BRÜCKNER, F. STOCK, A. SYMANCZYK, G. STANZL,

K. KUGLER, B. YENER-MARKSTEINER

The Excavation at Limyra/Lycia 2018: Preliminary Report

Alessandra RICCI

The Küçükyalı Arkeopark (Istanbul), 2016-2018: Excavation, Conservation, Cultural Heritage and Public Archaeology

Nergis GÜNSENIN

A Günsenin IV Amphora from Küçükyalı 


\title{
AKSEL TIBBET, UN COLLÈGUE À PORSUK ET DE PLUS UN AMI TRÈS CHER
}

\begin{abstract}
Nous avons fait connaissance en 1991, sur la terrasse de l'Institut français d'études anatoliennes, où une réception était organisée à l'occasion d'un colloque'. Aksel, en me présentant un plateau de petits-fours, m'a appris qu'il participait à la mission d'Olivier Pelon à Porsuk. Il m'a d'emblée demandé si j'avais conservé les relevés des poutres tombées dans la poterne et le « couloir coudé » des fortifications hittites dont il avait repris la fouille. Nous avons tout de suite sympathisé et, après des échanges de courriers et de plans, nous nous sommes retrouvés en 1992 à Porsuk avec la mission (Fig. 1).

Pendant vingt-deux ans, nous avons eu de nombreuses et fructueuses discussions sur son chantier, agrémentées de verres de thé servis par les mêmes ouvriers qu'Aksel reprenait d'une année sur l'autre. En fin de mission, il ne partait pas sans leur avoir offert quelques denrées alimentaires pour améliorer leur ordinaire. Il arrivait également avec des cadeaux : des livres pour les enfants du gardien qui font maintenant tous les deux de brillantes études supérieures. Nous restions en relation après les fouilles par d'innombrables échanges de courriers, en particulier lors de la préparation des
\end{abstract} rapports annuels pour le Ministère des Affaires étrangères et dans Anatolia Antiqua. Notre parfaite entente dans la compréhension et l'interprétation des vestiges du « chantier II » qu'il fouillait nous a donné l'idée de faire une communication en duo à la Rencontre de l'IFEA sur la Cappadoce méridionale, en hommage à Olivier Pelon, décédé de façon inattendue un mois plus tôt. L'article paru dans les Actes des $3^{e}$ Rencontres de l'IFEA ${ }^{2}$ témoigne de notre complicité.

Ce n'est pas seulement par relation professionnelle, mais par une profonde amitié qu'Aksel s'est totalement investi, en 2014, dans la préparation d'une exposition à la mémoire de mon père, Emmanuel Laroche, en relation avec un colloque sur «l'hittitologie aujourd'hui » ${ }^{3}$. Aksel s'est non seulement occupé de l'exposition,

*) UMR 7044, Archimède, Strasbourg.

1) Intitulé du colloque de 1991 : «Les grands ateliers d'Architecture dans le monde égéen du VI e siècle av. J.-C. ». Ma présentation portait sur les édifices de cette époque à Meydancıkkale.

2) Tibet, A. et Laroche-Traunecker, F. 2015 : «Les fortifications occidentales de Porsuk, restitution et modélisation des états les plus anciens », dans : Dominique Beyer, Olivier Henry et Aksel Tibet (éds.), La Cappadoce méridionale de la préhistoire à la période byzantine, Actes des $3^{e}$ Rencontres d'archéologie de l'IFEA, Istanbul 8-9 novembre 2012, Istanbul : 111-130.

3) L'idée et l'initiative de cette exposition commémorant le centenaire de la naissance d'Emmanuel Laroche sont dues à JeanFrançois Pérouse, alors directeur de l'IFEA. 


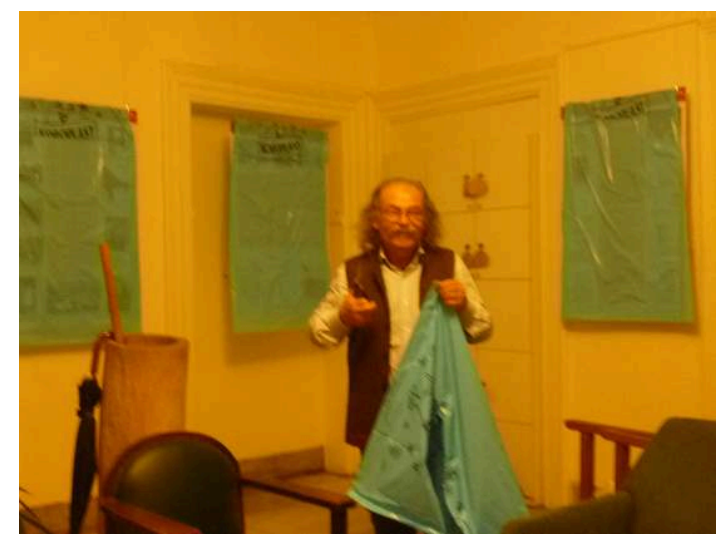

Fig. 2 : Aksel dans le hall d'entrée de l'IFEA, installant des panneaux sur la vie et l'œuvre d'Emmanuel Laroche (cliché Fr. Laroche-Traunecker, 2014).

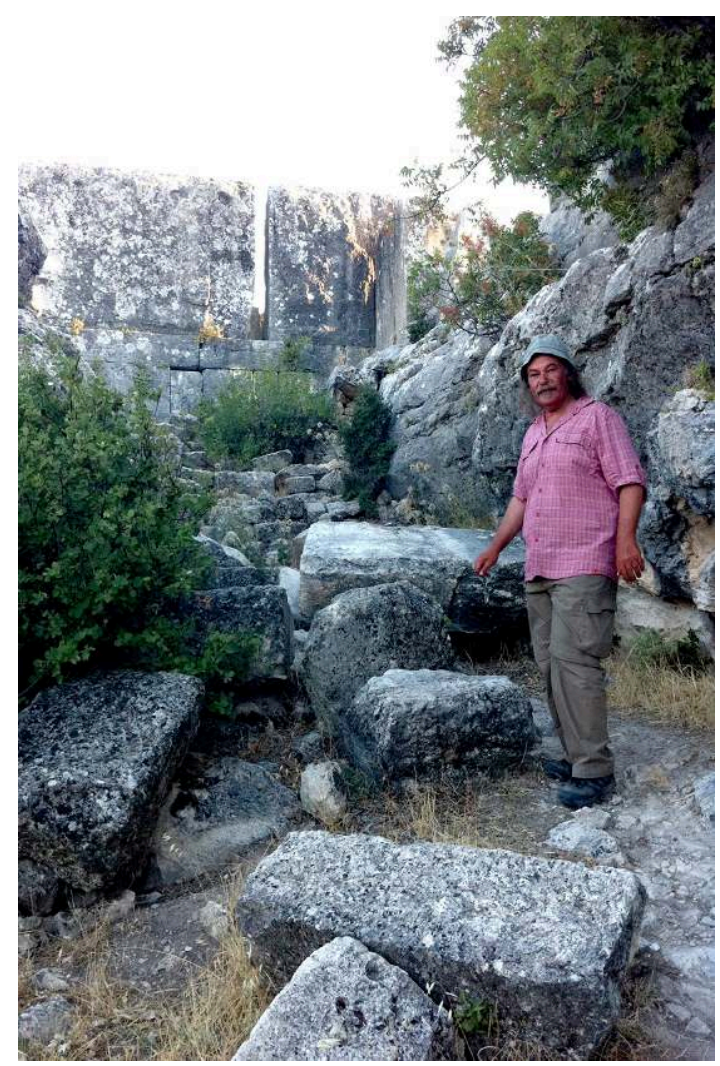

Fig. 4 : Aksel sur la rampe d'accès du site de Meydancık Kalesi, près de Gülnar (cliché Fr. Laroche-Traunecker, 2012).

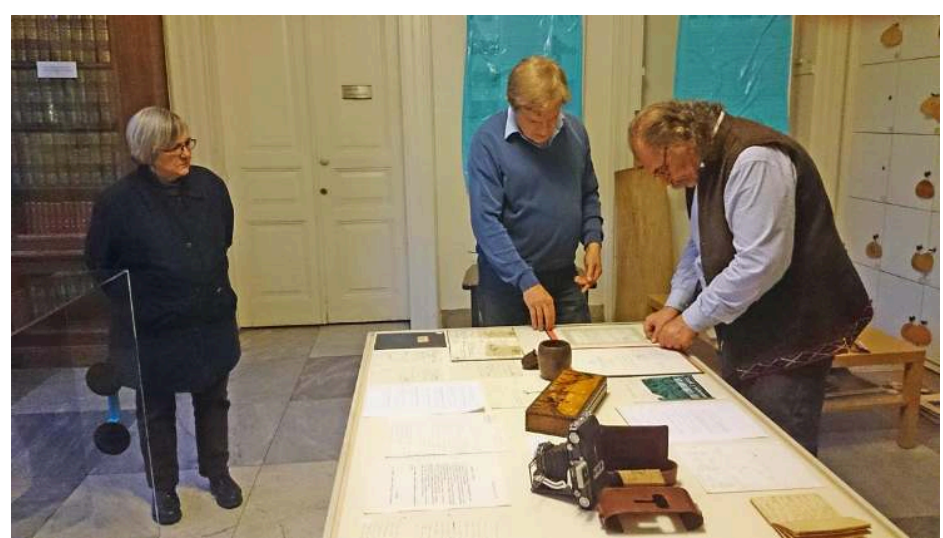

Fig. 3 : Aksel disposant des objets dans une vitrine d'exposition (cliché D. Laroche, 2014).

mais a de plus composé et édité une plaquette biographique. En arrivant dans le hall d'entrée de l'IFEA, avec mes deux frères venus également à Istanbul à cette occasion, nous avons trouvé Aksel sur une échelle en train d'accrocher les panneaux qu'il avait composés (Fig. 2). Nous avons assisté également à la disposition des documents dans les vitrines (Fig. 3). Aksel nous a réservé d'autres découvertes : de sympathiques petits restaurants et cafés du quartier où il nous a emmenés.

Sa collaboration amicale et désintéressée ne s'est pas arrêtée avec la fin de notre participation à la mission de Porsuk. En 2018, je lui avais fait part d'un projet de présentation du site de Meydancikkale à l'occasion d'une manifestation culturelle à Gülnar'. Très intéressé par ce site lorsque nous l'avions visité en 2013 avec l'équipe de Porsuk (Fig. 4), il m'a spontanément proposé de traduire en turc des textes ou des légendes de photographies si j'en avais besoin. Grâce à son aide et à ses conseils, j'ai pu composer plusieurs panneaux expliquant l'histoire du site et des fouilles.

Parallèlement à l'archéologie, Aksel s'adonnait à sa passion pour la musique. Tous ses amis savent qu'il animait une émission de musique classique à la radio le dimanche et qu'il participait à des concerts, parfois à la viole de gambe, plus souvent en tant que choriste. Cette dernière pratique me rappelle une anecdote qui l'avait beaucoup amusé. Lors de son séjour à Strasbourg en $2010^{5}$, après les réunions de travail, il allait visiter les églises de la ville et se renseigner sur leurs orgues. Un dimanche matin, il est arrivé tout émoustillé à la maison, revenant de la cathédrale où il avait assisté à une messe. Ayant

4) $3^{\text {e }}$ Manifestation internationale scientifique et culturelle de Gülnar.

5) Dominique Beyer avait organisé à Strasbourg un séminaire réunissant les membres de la mission de Porsuk pour faire le point sur l'état des connaissances, les dernières découvertes et proposer des projets à venir. 


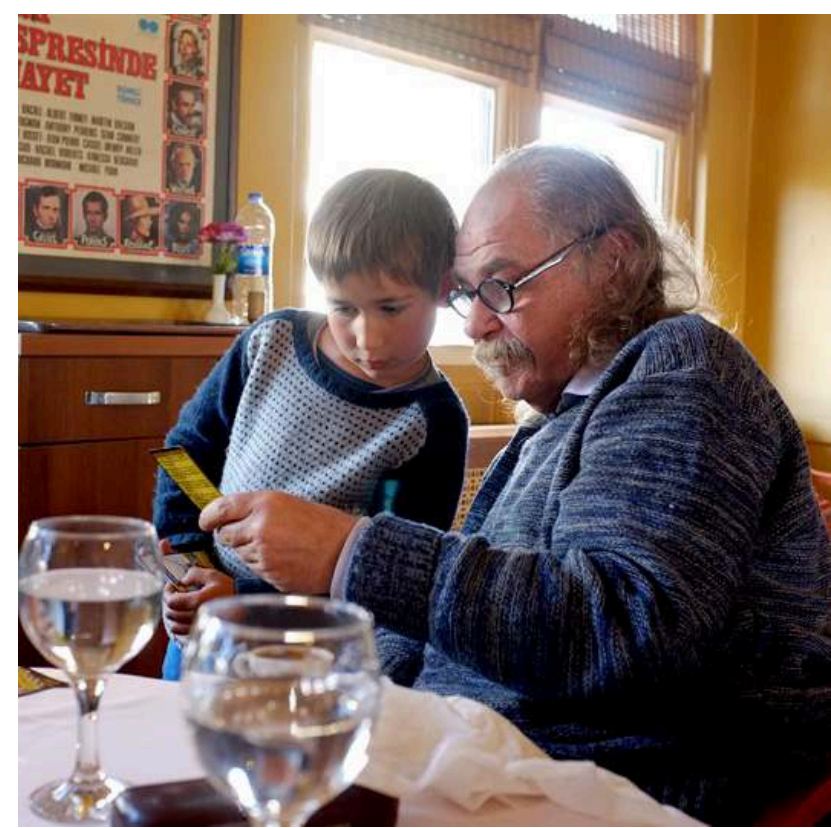

Fig. 5 : Aksel examinant des fiches documentaires avec Lucien, 7 ans (cliché J. Traunecker, 2017).

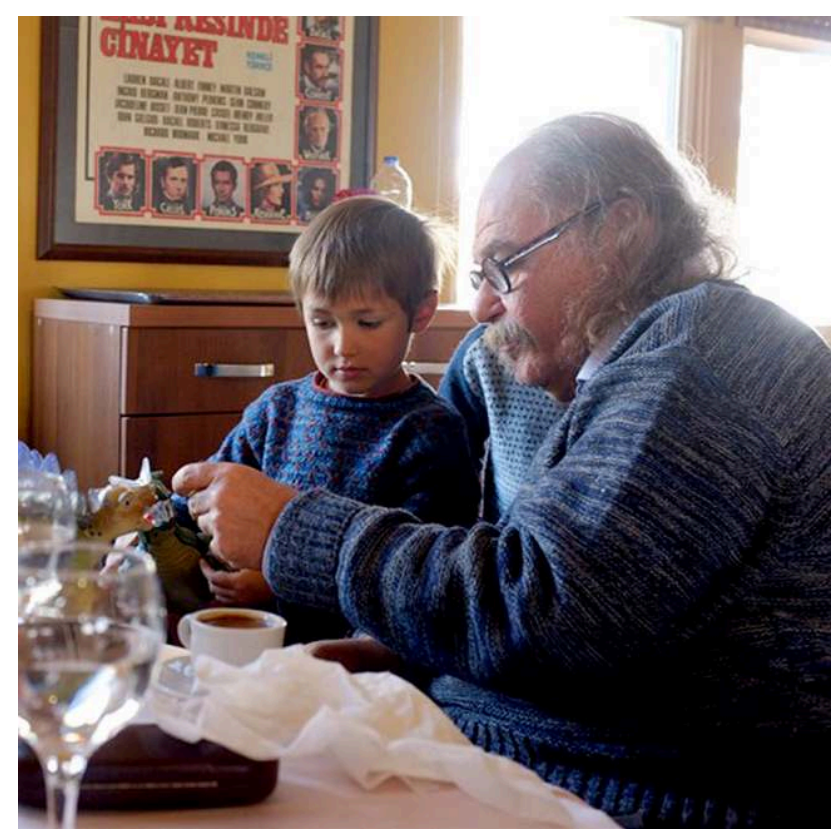

Fig. 6 : Aksel examinant un dinosaure prêté par Antoine, 5 ans (cliché J. Traunecker, 2017).

mêlé sa voix aux chants, debout à côté d'une dame âgée, celle-ci l'a serré dans ses bras à la fin et l'a embrassé chaleureusement en lui disant :

«Merci, Monsieur, quel bonheur de vous avoir entendu chanter avec une telle ferveur! »

Je termine par une vision inhabituelle d'Aksel, avec des photographies qui démentent sa réputation de ne pas beaucoup s'intéresser aux enfants (Fig. 5 et 6). En septembre 2017, je n'ai passé que trois jours à Istanbul pour présenter la ville à mon fils aîné ${ }^{6}$, sa compagne et leurs enfants. Bien que disposant de très peu de temps, je tenais beaucoup à rencontrer Aksel. Il nous a tous invités au restaurant où il a prêté une oreille très attentive aux histoires de dinosaures racontées par les enfants et a longuement discuté avec eux. C'est un des moments privilégiés passés avec Aksel que je n'oublierai jamais.

Françoise Laroche-Traunecker, juillet 2019

6) Mon fils connaissait déjà Istanbul et Aksel, puisqu'il avait participé à la mission de 2004 à Porsuk. 


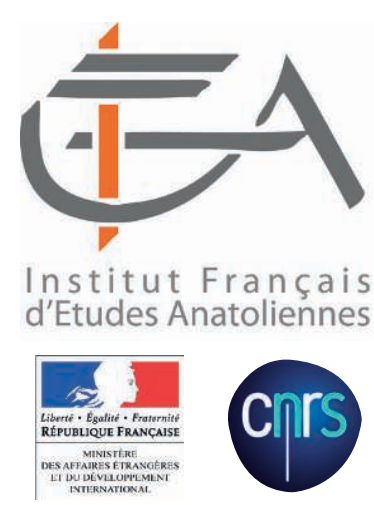

Figurine d'homme en terre cuite (Louvre, Tarse 314) (C) 2016 Réunion des musées nationaux / Tony Querrec. 